\title{
Breached Pairing Superfluidity at Finite Temperature and Density
}

\author{
Jinfeng Liao and Pengfei Zhuang \\ Physics Department,Tsinghua University,Beijing 100084
}

A general analysis on Fermion pairing at finite temperature and density between different species with mismatched Fermi surfaces is presented. Very different from the temperature effect of BCS phase, the recently found breached pairing phase resulted from density difference of the two species lies in a region with calabash-like shape in the $T-\mu$ plane, and the most probable temperature for the new phase's creation is finite but not zero.

PACS numbers: 74.20.-z, 03.75.Kk, 12.38.-t

The Fermion pairing between different species with mismatched Fermi surfaces, which has already been discussed many years ago in investigating superconducting metal or B state of liquid ${ }^{3} \mathrm{He}$ in a magnetic field [1,2], prompted recently new interest in both theoretical and experimental studies. In high energy physics the strong interacting matter at high baryon densities described by Quantum Chromodynamics (QCD) is a color superconducting phase, in which quarks of different colors pair with each other due to attraction in color anti-triplet channel $[3,4]$. The corresponding physical systems may be found in compact stars [5]. In non-relativistic case the study is related to the challenging goal of observing the BCS transition in trapped Fermionic atoms [6-9], the electrons in solid distributed in two different bands [6,9], and the neutron-proton pairing in isospin asymmetric nuclear matter [10].

When the two Fermions have the same Fermi surface, the pairing phenomena is the well-known BCS mechanism [11]: At low temperature but high density, Fermionic matter with a sharp and high Fermi surface is unstable under attractive interaction(even very weak), a BCS paired state is favored instead. However, under some physical constraints the two Fermions may come from different Fermi surfaces. In atom traps, there can be two different hyperfine states of the same atom $\left({ }^{6} \mathrm{Li}\right.$ or ${ }^{40} K$ ) which serve as two attracting species of fermions, and even mixture of two different Fermionic atoms could be realized [6-9]. In QCD matter at moderate baryon density (below the critical Color-Flavor-Locking density) which is considered to be relevant for understanding neutron stars, the mass difference between light quarks ( $u$ and d) and strange quark can not be neglected, and therefore they have different Fermi momenta [12]. When chiral symmetry restoration at finite density is considered [13], the mass difference becomes more significant, and the mismatch will be enhanced. The mismatch happens even for the pairing between the light quarks when charge neutrality is taken into account [14].
A well-known pairing mechanism of Fermions from mismatched Fermi surfaces is the LOFF state [1]. Different from a BCS pair which has zero total momentum, a LOFF pair has finite total momentum. An analogue of this idea in dense QCD matter is the crystalline color superconductivity [15]. Recently a spatially uniform mechanism is proposed [6] which leads to a breached pairing superfluidity (BP) [9] with coexistence of pairing state at lower and higher Fermi surfaces and particle state in between, when the densities of the two species differ from each other. This new phase is argued to be more favored than the LOFF phase for certain region of parameters.

It is well-known that with increasing temperature of the system the superfluidity gap in a BCS state drops down monotonously and the region of superfluidity phase in the temperature-density plane is reduced monotonously too [16]. What is the temperature effect of a system in a BP state and what is the difference from that in a BCS state? In this letter, we present a general analysis on spatially uniform pairing state with mismatched Fermi surfaces at finite temperature and density. We will discuss the stability of BP state induced by mass difference of the two species, and calculate the temperature behavior of the superfluidity gap and the phase diagram for a system with two species of different densities.

We start with a system containing two species of fermions represented by $a$ and $b$. The interaction can be modelled by a four-fermion point coupling, which is appropriate for both trapped Fermionic atoms and dense quark systems [3,6-9]. Since our purpose is a general analysis for pairing phenomena, we neglect inner structures like spin, isospin, flavor and color, which are important and bring much abundance while are not central for pairing. We write down the following Hamiltonian,

$$
\hat{\mathcal{H}}=\frac{1}{V} \sum_{\vec{p}}\left(\epsilon_{p}^{a} \hat{a}_{\vec{p}}^{\dagger} \hat{a}_{\vec{p}}+\epsilon_{p}^{b} \hat{b}_{\vec{p}}^{\dagger} \hat{b}_{\vec{p}}\right)-\frac{g}{V^{2}} \sum_{\vec{p}, \vec{q}} \hat{a}_{\vec{p}}^{\dagger} \hat{b}_{-\vec{p}}^{\dagger} \hat{b}_{-\vec{q}} \hat{a}_{\vec{q}}
$$

where $\mathbf{p}$ and $\mathbf{q}$ are momenta of the species, $\hat{a}, \hat{b}, \hat{a}^{\dagger}$ and $\hat{b}^{\dagger}$ are the annihilation and creation operators, the coupling constant $g$ is positive to keep the interaction attractive, $V$ is the system volume and in continuous limit we simply replace $\frac{1}{V} \sum_{\vec{p}}$ with $\int \frac{d^{3} \mathbf{p}}{(2 \pi)^{3}}$, and the effective particle energies $\epsilon_{p}^{a, b}$ are $\sqrt{p^{2}+m_{a, b}^{2}}-\mu_{a, b}$ in relativistic case and $\frac{p^{2}}{2 m_{a, b}}-\mu_{a, b}$ in non-relativistic case. The Fermi momenta $p_{F}^{a, b}$ determined by $\epsilon_{p}^{a, b}=0$ are controlled by the particle masses $m_{a, b}$ and the chemical potentials $\mu_{a, b}$. Whether 
$\mu_{a, b}$ can be used as free parameters depends on the physical system we discuss. When the densities of the species are restricted by some physical constraint like fixed overall particle density or fixed relative particle density, $\mu_{a, b}$ are not fully free and should be adjusted to satisfy the constraint. In fact, it is the density constraint which brings nontrivial BP state, as pointed out in [6-9] and will be clearly shown below.

We introduce in the light of Mean-Field an order parameter $\Delta=\frac{g}{V} \sum_{\vec{p}}<\hat{a}_{\vec{p}}^{\dagger} \hat{b}_{-\vec{p}}^{\dagger}>$ and make it real by proper choice of phase factors of the creation operators, which allows us to diagonalize the Hamiltonian into

$$
\hat{\mathcal{H}}_{\text {diag }}=\frac{1}{V} \sum_{\vec{p}}\left(E_{p}^{A} \hat{A}_{\vec{p}}^{\dagger} \hat{A}_{\vec{p}}+E_{p}^{B} \hat{B}_{\vec{p}}^{\dagger} \hat{B}_{\vec{p}}+\epsilon_{p}^{b}\right)+\frac{\Delta^{2}}{g}
$$

after a Bogliubov transformation from Fermions $a$ and $b$ into quasi-Fermions $A$ and $B$ with annihilation and creation operators $\hat{A}, \hat{B}, \hat{A}^{\dagger}, \hat{B}^{\dagger}$ and quasi energies

$$
E_{p}^{A, B}=\epsilon_{p}^{-} \pm \sqrt{\epsilon_{p}^{+2}+\Delta^{2}}
$$

with

$$
\epsilon_{p}^{ \pm}=\frac{\epsilon_{p}^{a} \pm \epsilon_{p}^{b}}{2}
$$

The dispersions for particles $a, b$ and quasi-particles $A, B$ in relativistic case are shown in Fig.(1). With the interest in mismatched Fermi momenta induced by mass difference and density difference, a remarkable feature is that $E_{p}^{A, B}$ can cross zero between the particle Fermi momenta $p_{F}^{a, b}$. A simple algebra calculation shows that the condition for $E_{p}^{A(B)}$ to cross zero is the constraint $\Delta<\Delta_{c}$ for the gap. In relativistic case with the approximation of $p_{F}^{a, b}>>m_{a, b}$, the critical gap is

$$
\Delta_{c}=\frac{\sqrt{p_{F}^{a} \cdot p_{F}^{b}}}{2 \sqrt{\mu_{a} \mu_{b}}}\left|p_{F}^{a}-p_{F}^{b}\right|
$$

in non-relativistic case one has exactly

$$
\Delta_{c}=\frac{p_{F}^{a}+p_{F}^{b}}{4 \sqrt{m_{a} m_{b}}}\left|p_{F}^{a}-p_{F}^{b}\right|
$$

For convenience, we introduce momentum interval $I \equiv$ $\left\{\vec{p}\left|p_{-}<\right| \vec{p} \mid<p_{+}\right\}$with $p_{ \pm}$the solutions of $E_{p}^{A(B)}=0$.

Whether $E_{p}^{A(B)}$ crosses zero along the momentum axis plays a crucial role in pairing with mismatched Fermi surfaces. $E_{p}^{A(B)}=0$ means directly gapless excitation of quasi-particles in a superfluidity phase observed in [4,6-9,17]. We see from Fig.(1) large mismatch and small gap leave space for gapless phenomena. If there is no mismatch, there is no place for gapless excitation.

In order to understand the coexistence of pairing state and particle state when the gapless excitation region $I$ is not empty, we write down the ground state of the system in terms of the zero quasi-particle state $\mid 0_{p}^{A(B)}>$ and one quasi-particle state $\mid 1_{p}^{A(B)}>$ at zero temperature,
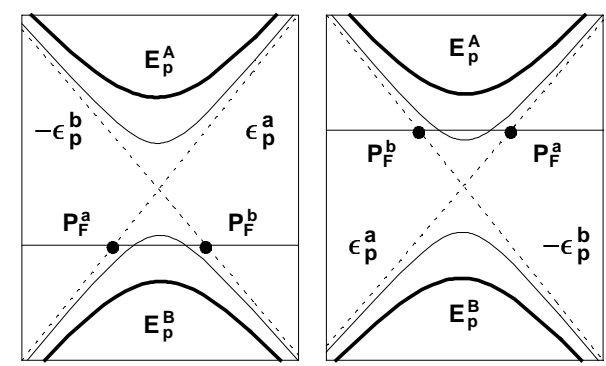

FIG. 1. Dispersions of particles (dashed lines) and quasi-particles (solid lines). The thick and thin solid lines correspond to $\Delta<\Delta_{c}$ and $\Delta>\Delta_{c}$, respectively. The left panel is for $E_{p}^{B}=0$, and the right panel is for $E_{p}^{A}=0$.

$$
\begin{aligned}
\mid g>=\prod_{\vec{p}} & \left(\theta\left(E_{p}^{A}\right) \theta\left(-E_{p}^{B}\right) \mid 0_{p}^{A}, 1_{p}^{B}>\right. \\
& \left.+\theta\left(-E_{p}^{A}\right)\left|1_{p}^{A}, 1_{p}^{B}>+\theta\left(E_{p}^{B}\right)\right| 0_{p}^{A}, 0_{p}^{B}>\right)
\end{aligned}
$$

where $\theta(x)$ is a step function. With the help of the Bogliubov transformation the ground state can be expressed in the space of the original Fermions $a$ and $b$,

$$
\begin{aligned}
\mid g>= & \prod_{\vec{p} \notin I}\left(\cos \theta_{p}-\sin \theta_{p} \hat{a}_{\vec{p}}^{\dagger} \hat{b}_{-\vec{p}}^{\dagger}\right) \mid 0_{p}^{a}, 0_{p}^{b}>\times \\
& \prod_{\vec{p} \in I}\left(\theta\left(p_{F}^{a}-p_{F}^{b}\right) \hat{a}_{\vec{p}}^{\dagger}+\theta\left(p_{F}^{b}-p_{F}^{a}\right) \hat{b}_{-\vec{p}}^{\dagger}\right) \mid 0_{p}^{a}, 0_{p}^{b}>.
\end{aligned}
$$

The above state tells us clearly the breached pairing phenomena. If neither $E_{p}^{A}$ nor $E_{p}^{B}$ crosses zero, namely without gapless excitation, the species $a$ and $b$ are symmetrically and partially occupied and paired, the system is in a BCS pattern. If one can find roots $p_{ \pm}$from $E_{p}^{A(B)}=0$, there exists a momentum interval $I$ in which one specie is fully occupied and the other is fully empty. The pairing occurs only in the region around the lower Fermi surface and the region around the higher Fermi surface, the two regions are separated by the gapless excitation region $I$. This means a new phase - the breached pairing superfluidity proposed in [9]. The observed gapless phenomena $[4,6-8,17]$ all fall into the BP phase, which is characterized by two universal features, pairing breached by single occupation and simultaneous gapless and gaped components.

We now turn to thermodynamics. From the Hamiltonian (2) in quasi-Fermion representation the grand potential is easily expressed as

$$
\begin{aligned}
\Omega\left(T, \mu_{a}, \mu_{b}, \Delta\right) & =\frac{\Delta^{2}}{g}-\frac{1}{V} \sum_{\vec{p}}\left[\left(\frac{E_{p}^{A}}{2}+T \operatorname{Ln}\left(1+e^{-E_{p}^{A} / T}\right)\right)\right. \\
& \left.+\left(\frac{E_{p}^{B}}{2}+T \operatorname{Ln}\left(1+e^{-E_{p}^{A} / T}\right)\right)-\epsilon_{p}^{+}\right]
\end{aligned}
$$


With given $\Omega$ as a function of temperature, chemical potentials and gap parameter, all other thermodynamical quantities can be immediately obtained and its minimum gives the gap equation for solving $\Delta$

$$
\Delta \cdot\left[\frac{2}{g}-\frac{1}{V} \sum_{\vec{p}} \frac{f\left(E_{p}^{B}\right)-f\left(E_{p}^{A}\right)}{\sqrt{\epsilon_{p}^{+2}+\Delta^{2}}}\right]=0
$$

with the Fermi-Dirac distribution $f(x)=\frac{1}{e^{x / T}+1}$.

To do numerical calculation and present quantitative results we consider in the following only relativistic case. The calculation can straightforwardly be extended to non-relativistic case. Since the four-Fermion interaction is not renormalizable, one must use a momentum cutoff $\Lambda$ to avoid divergence. We choose $\Lambda=600 \mathrm{MeV}$ in our numerical calculation. The mismatch of the Fermi surfaces can be induced by either mass difference or density difference of the two species. We now solve the gap equation (10) at finite temperature in the two cases.

Mass Difference - We consider a system with $\mu_{a, b}=$ $\mu$ but $m_{a} \neq m_{b}$ to study the effect of mass difference. For given $m_{a}, m_{b}, \mu$ and $T$ one can define two couplings $g_{c 1}<g_{c 2}$, corresponding to the critical conditions to have a BP state and a BCS state, respectively,

$$
\begin{aligned}
& g_{c 1}=\left.\left[2 / \frac{1}{V} \sum_{\vec{p}} \frac{f\left(E_{p}^{B}\right)-f\left(E_{p}^{A}\right)}{\sqrt{\epsilon_{p}^{+2}+\Delta^{2}}}\right]\right|_{\Delta \rightarrow \Delta_{c}}, \\
& g_{c 2}=\left.\left[2 / \frac{1}{V} \sum_{\vec{p}} \frac{f\left(E_{p}^{B}\right)-f\left(E_{p}^{A}\right)}{\sqrt{\epsilon_{p}^{+2}+\Delta^{2}}}\right]\right|_{\Delta \rightarrow 0} .
\end{aligned}
$$

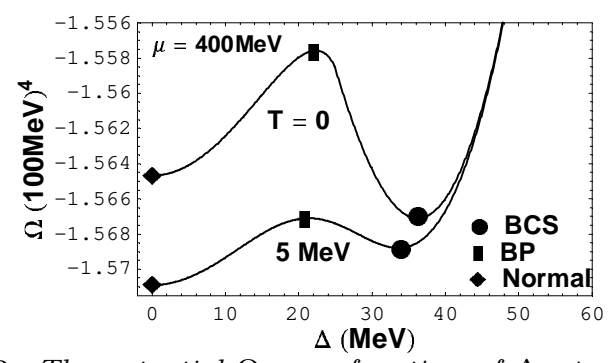

FIG. 2. The potential $\Omega$ as a function of $\Delta$ at given $T, \mu$ with $m_{a}=5 \mathrm{MeV}, m_{b}=200 \mathrm{MeV}$, and $g=50 \mathrm{GeV}^{-2}$.

For $g<g_{c 1}$ the only solution of (10) is $\Delta=0$ which means a normal phase without pairing. For $g>g_{c 2}$ there is a stable BCS solution and $\Delta=0$ becomes unstable. When $g$ is in the region $g_{c 1}<g<g_{c 2}$, there are three solutions of (10): a normal solution $\Delta=0$, a BCS solution $\Delta>\Delta_{c}$ and a BP solution $\Delta<\Delta_{c}$. However, only the BCS solution is the global minimum of $\Omega$, the $\mathrm{BP}$ solution is unstable because it doesn't satisfy the stable condition $\partial^{2} \Omega / \partial \Delta^{2}>0$, and the normal solution is metastable. This is shown clearly in Fig.(2). When the temperature increases, the normal solution becomes stable and the BCS solution changes to be metastable. In any case the BP solution can not be stable. When $T$ exceeds some critical value, the unstable BP state even disappears. Therefore, the stability analysis rules out the possibility to create a BP phase by mass difference.

Density Difference — From the thermodynamic potential (9) it is easy to obtain the particle densities

$$
\begin{aligned}
& n_{a}=\frac{1}{2 V} \sum_{\vec{p}} {\left[\left(1+\frac{\epsilon_{p}^{+}}{\sqrt{\epsilon_{p}^{+2}+\Delta^{2}}}\right) f\left(E_{p}^{A}\right)\right.} \\
&\left.+\left(1-\frac{\epsilon_{p}^{+}}{\sqrt{\epsilon_{p}^{+2}+\Delta^{2}}}\right) f\left(E_{p}^{B}\right)\right], \\
& n_{b}=\frac{1}{2 V} \sum_{\vec{p}}\left[-\left(1-\frac{\epsilon_{p}^{+}}{\sqrt{\epsilon_{p}^{+2}+\Delta^{2}}}\right) f\left(E_{p}^{A}\right)\right. \\
&\left.-\left(1+\frac{\epsilon_{p}^{+}}{\sqrt{\epsilon_{p}^{+2}+\Delta^{2}}}\right) f\left(E_{p}^{B}\right)+2\right] .
\end{aligned}
$$

We consider a system with fixed relative density

$$
\frac{n_{b}}{n_{a}}=\lambda
$$

To satisfy this density constraint, namely a charge conservation such as electronic charge with $a, b$ carrying charge number $-\lambda,+1$, respectively, the chemical potentials should be adjusted to be $\mu_{a}=\mu-\lambda \delta \mu$ and $\mu_{b}=\mu+\delta \mu$, where $\mu$ corresponds to the total number density since $n_{a}+n_{b}=-\partial \Omega / \partial \mu$. Unlike the case of mass difference where the chemical potentials are free and $\Delta$ is purely determined by the gap equation, here only $\mu$ is free and $\Delta$ and $\delta \mu$ are coupled to each other through (10) and (13).

For $\lambda=1$ the system can be in a BCS state, while for $\lambda \neq 1$ there will be some particles left after the pairing, the system must be in a BP state if pairing occurs. We first calculate $\Delta$ as a function of $\mu$ for $\lambda=2$ at given temperature. We choose the parameters $m_{a}=m_{b}=5 \mathrm{MeV}$, and $g=80 \mathrm{GeV}^{-2}$ in the following numerical calculation. Introducing mass difference in calculation brings only quantitative changes. The value of $g$ needed to produce a BP decreases fast with increasing momentum cutoff $\Lambda$, and taking into account all inner degrees of freedom like spin, isospin, color and flavor will reduce the coupling by order of magnitude. From Fig.(3) a BP phase exists in a relatively narrow region, compared with a BCS state. The explanation for the existence of the higher critical chemical potential is that when $\mu$ is high enough the mismatch of the two Fermi surfaces is too large for the two species to pair at given coupling.

In a BCS state it is well-known that the gap and the region of superfluidity decrease monotonously with increasing temperature. However, the case in a BP state is very different. With increasing temperature, the gap 
firstly goes up and then drops down, and correspondingly the region firstly expands and then contracts. The temperature related to the maximum gap and the maximum region is a finite value but not zero. We can understand this feature in the following way. When the two Fermi surfaces coincide, the temperature deforms and lowers the Fermi surface and then the gap decreases. In the case of mismatch, the two different sharp Fermi surfaces are deformed and lowered by the temperature on one hand, but the expansion of the two distributions make the two species much closer in the phase space and then much easier to pair on the other hand. The former temperature effect reduces the gap but the latter effect favors the gap. The competition between the former and the latter effects controls the temperature dependence of the amplitude and the region of superfluidity.

The behavior of the gap as a function of temperature at fixed chemical potential for different relative density is shown in Fig.(4). At $\lambda=1$, the gap drops down monotonously due to disorder brought in by increasing temperature, a standard characteristic of BCS phase. For $\lambda \neq 1$, we see again the amazing temperature behavior: The gap first increases to a maximum value and then reduces rapidly to zero with increasing temperature. While the density difference $\lambda \neq 1$ is the prerequisite for a $\mathrm{BP}$ phase, the amplitude and the region of the gap decreases with increasing $\lambda$.

We further give in Fig.(5) the phase diagram in $T-\mu$ plane for the BP phase with $\lambda=2$ and compare it with the BCS phase diagram with $\lambda=1$. The calabash-like phase transition line from normal state to BP state manifests the competition between the two opposite temperature effects on the mismatched Fermi surfaces, discussed above. The most probable temperature for producing a $\mathrm{BP}$ state is not zero but a finite value.

In summary, we have presented a general analysis on Fermion pairing between different species with mismatched Fermi surfaces under the assumption of spatial uniform, and investigated especially the temperature behavior of the new BP phase. From the stability analysis the mass difference can not create a stable BP state. In the case of fixed relative density $n_{b} / n_{a} \neq 1$ the temperature effect not only deforms and reduces the mismatched Fermi surfaces which leads to the usual suppression of the gap, but also make the overlap region of the two species even wider which favors the condensate. The competition of the two opposite temperature effects results in a calabash-like phase transition line from normal to BP states. The temperature corresponding to the largest gap and the largest region of superfluidity is finite but not zero. Due to this novel temperature effect, we think that trapping different Fermionic atoms with different densities at controlled finite temperature may be a feasible way to obtain a $\mathrm{BP}$ phase.

We are grateful to Dr. M. Huang for her stimulating discussions. The work was supported in part by the
NSFC under contract numbers 19925519, 10135030 and 10105005.

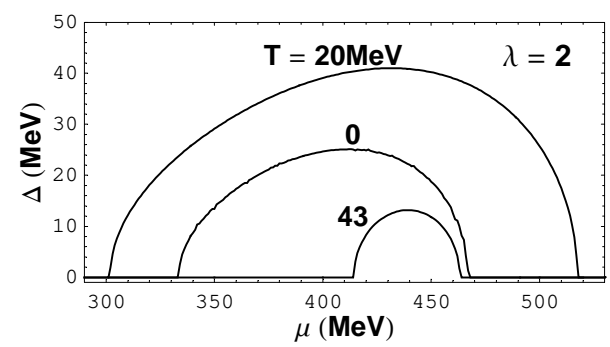

FIG. 3. The gap parameter as a function of $\mu$ at fixed relative density $\lambda=2$ for different temperature.

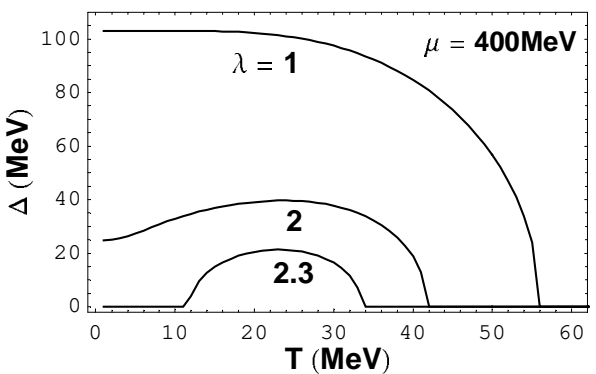

FIG. 4. The gap parameter as a function of $T$ at fixed chemical potential for different relative density.

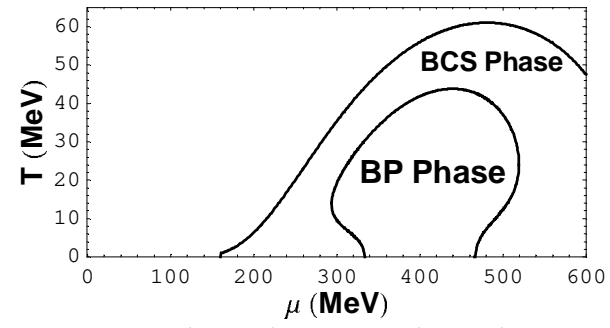

FIG. 5. The $B C S(\lambda=1)$ and $B P(\lambda=2)$ phase transition lines in $T-\mu$ plane.

[1] A. I. Larkin and Yu. N. Ovchinnikov, Sov. Phys. JETP 20(1965) 762; P. Fulde and R. A. Ferrell, Phys. Rev. 135(1964) A550.

[2] A. Leggett, Rev. Mod. Phys. 41(1975) 331.

[3] For reviews, see K. Rajagopal and F. Wilczek, hepph/0011333; M. Alford, Ann. Rev. Nucl. Part. Sci. 51(2001) 131; T. Schäfer, hep-ph/0304281.

[4] I. Shovkovy and M. Huang, hep-ph/0302142.

[5] N. K. Glendenning, Compact Stars - Nuclear Physics, particle Physics, and General Relativity, Springer (2000). 
[6] W. V. Liu and F. Wilczek, Phys. Rev. Lett. 90(2003) 047002.

[7] S. Wu and S. Yip, Physical Review A67 (2003) 053603.

[8] W. V. Liu and F. Wilczek, cond-mat/0304632.

[9] E. Gubankova, W. V. Liu and F. Wilczek, hep$\mathrm{ph} / 0304016$.

[10] A. Sedrakian and U. Lombardo, Phys. Rev. Lett. 84(2000) 602; U. Lombardo, P. Noziéres, P. Schuck, H.J. Schulze, and A. Sedrakian, Phys. Rev. C 64(2001) 064314.

[11] J. Bardeen, L. N. Cooper, and J. R. Schrieffer, Phys. Rev. 108(1957)1175.

[12] T. Schäfer and F. Wilczek, Phys. Rev. D 60(1999)074014; M. Alford, J. Berges and K. Rajagopal, Nucl. Phys. B $\mathbf{5 5 8 ( 1 9 9 9 ) 2 1 9 .}$

[13] M. Buballa and M. Oertel, Nucl. phys. A 703(2002)481.

[14] K. Rajagopal and F. Wilczek, Phys. Rev. Lett. 86(2001) 3492; A. W. Steiner, S. Reddy and M. Prakash, Phys. Rev. D 66(2002) 094007; M. Alford and K. Rajagopal, JHEP 0206(2002) 031; F. Neumann, M. Buballa and M. Oertel, Nucl. phys. A 714(2003) 481; M. Huang, P. Zhuang and W. Chao, Phys. Rev. D 67(2003) 065015.

[15] J. Kundu and K. Rajagopal, Phys. Rev. D 65(2002) 094022; J. Bowers and K. Rajagopal, Phys. Rev. D 66(2002) 065002.

[16] J. Berges, K. Rajagopal, Nucl. phys. B 538(1999) 215; J. Liao, Diploma thesis, Tsinghua University, 2001.

[17] M. Alford, J. Berges and K. Rajagopal, Phys. Rev. Lett. 84(2000) 598. 